\title{
Undetected pulmonary Artery Aneurysms Secondary to Prolonged Right-sided Endocarditis in a Patient with Corrected Tetralogy of Fallot
}

Vasiliki Katsiva ${ }^{1}$, Charalampopoulos Georgios ${ }^{2}$, Stefanidis Alexandros ${ }^{1}$, Keramida Kalliopi ${ }^{3 *}$, Leventogiannis Georgios $^{1}$, Vallianatou Xanthippi ${ }^{1}$ and Kelekis Nikolaos ${ }^{4}$

${ }^{1}$ General Hospital of Nikea-Pireus, Greece

${ }^{2}$ University General Hospital, Greece

${ }^{3}$ Hammersmith Hospital, UK

${ }^{4}$ National and Kapodistrian University of Athens, Greece

Submission: October 07, 2016; Published: November 03, 2016

*Corresponding author: Keramida Kalliopi, Pavlou Mela 4, Ilioupolis, Athens, 16346, Greece, Tel: +306947996393; +447511583580; Fax:

+302109737973; Email: keramidakalliopi@hotmail.com

\section{Abstract}

Mycotic pulmonary artery aneurysms are a rare complication of right-sided endocarditis most often associated with congenital heart disease or intravenous drug addiction. We report a case of undetected mycotic pulmonary artery aneurysms secondary to prolonged rightsided endocarditis in a 25-years old patient with corrected tetralogy of Fallot/pulmonary atresia. An attempt to explain the delayed diagnosis of endocarditis with subsequent mycotic aneurysms formation is made and the clinical and imaging findings of this rare complication during a long-term follow up are presented, contributing to our knowledge on the natural history of this disease.

Keywords: Mycotic aneurysms; Tetralogy of Fallot /pulmonary atresia; Right heart endocarditis; Kocuria varians

Abbreviations: CT: Computed Tomography; TTE: Trans Thoracic Echocardiogram; MRI: Magnetic Resonance Imaging; MRA: Magnetic Resonance Angiography; PAAs: Pulmonary Artery Aneurysms

\section{Introduction}

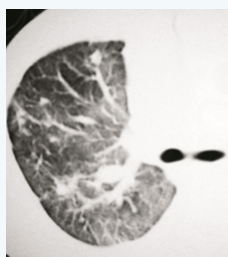

$(1 \mathrm{~A})$

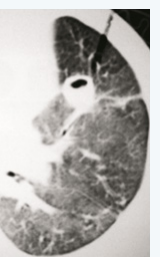

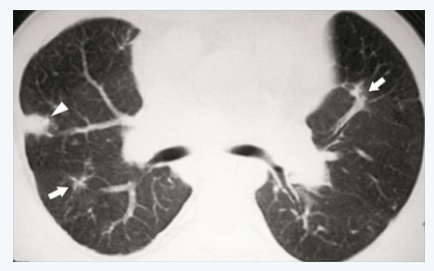

(1B)

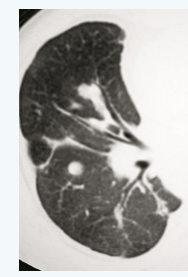

$(1 \mathrm{C})$
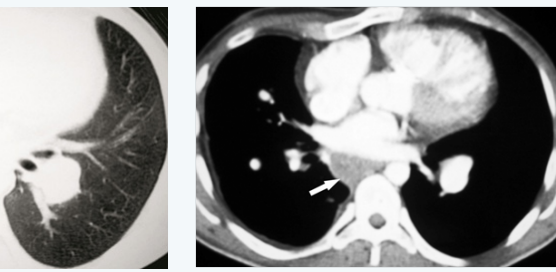

(1D)

Figure 1: CT scans obtained one year before, during admission for hemoptysis. Multiple, bilateral, nodular infiltrates (one cavitating) are shown:

(A): After two months, most of them regressed (arrows) but a new one appeared (arrowhead).

(B): One month later, contrast enhanced CT scan shows two aneurysms of segmental arterial branches in the middle and right lower lobe that went undetected.

(C) and (D): The diameter of the aneurysms (the others not shown) ranged from 7-10 mm. pulmonary infiltrates have been cleared but mediastinal lymphadenopathy persists (arrow). 
A 25-years old man, with corrected tetralogy of Fallot/ pulmonary atresia at the age of 5 years and subsequent angioplasty for pulmonary artery conduit stenosis at the age of 20 years, presented with hemoptysis, low grade fever, signs of right heart failure and splenomegaly. During last five years and shortly after the conduit angioplasty he also referred multiple episodes of hemoptysis. In-hospital serial chest computed tomography (CT) scans during each admission (Figure 1) showed multiple migrating infiltrates and nodules and mediastinal lymph adenopathy with only temporary regression after short term antibiotic therapy. Considering retrospectively previous chest CT scans, we noticed the presence of multiple, bilateral pulmonary artery aneurysms (PAAs), unchanged in number and size (7-10mm in diameter) for the last two years before current admission (Figure 1). The aneurysms were not apparent on the initial evaluation (but contrast enhanced studies were missing at that time). Additionally, despite the broad diagnostic workup performed in each admission time, which also included cardiac echocardiography, cardiac magnetic resonance imaging (MRI) and bronchoscopy, the cause of hemoptysis remained unclear.

The patient underwent a chest high resolution CT scan (not shown) revealing patchy infiltrates and a trans thoracic echocardiogram (TTE). The pulmonary valve was highly echogenic with significant stenosis and regurgitation indicating major degeneration. Furthermore, from the supra sternal view an oscillating nodular mass attached inside the conduit was demonstrated indicating possible vegetation or thrombus (Figure 2). However, a high index of suspicion for endocarditis was justified since the mass was attached in the physically damaged endothelium by the high turbulent flow of the stenotic right ventricular outflow tract. Trans esophageal echo was not performed since it could not give significant additional information.

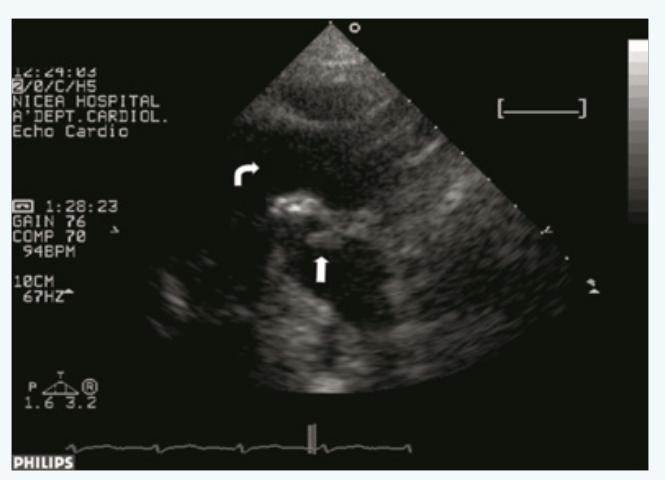

Figure 2: Transthoracic echocardiogram, suprasternal projection Oscillating nodular mass (arrow) inside the graft conduit indicating possible vegetation. Aortic arch (curved arrow).

Blood cultures were taken and intravenous antibiotic treatment for endocarditis was initiated with vancomycin and gentamycin and then with penicillin $\mathrm{G}$ after the detection of Kocuria Varians. Cardiac MRI and magnetic resonance angiography (MRA) of the pulmonary vessels revealed graft conduit stenosis, pulmonary valve regurgitation, impaired right ventricular function and multiple aneurysms located in the pulmonary artery segmental branches of both lower lobes and right middle lobe (Figures $3 \& 4$ ). Since last CT evaluation (one year before), the aneurysms remained unchanged in number and size except for the one in the middle lobe which was found to have been almost duplicated.

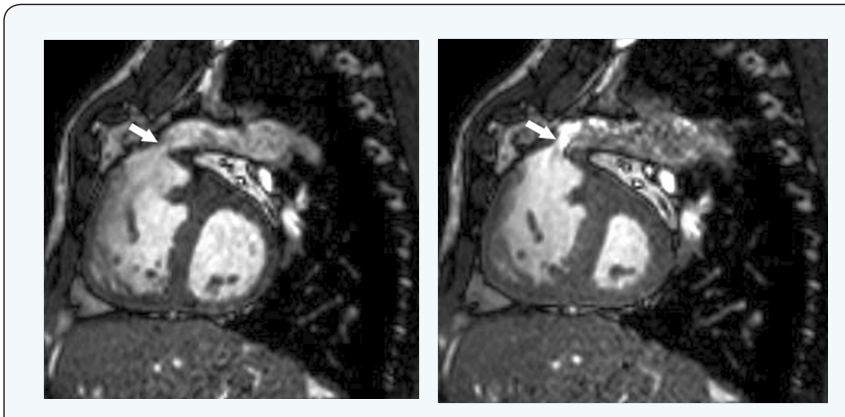

Figure 3: Cardiac MRI. Cinegradient echo images of the right ventricular outflow tract (RVOT) during diastole.

(A) And systole.

(B) Show a high grade stenosis in the pulmonary artery graft origin as indicated by the reduced diameter of the RVOT.

(A) (Arrow) and the high velocity jet flow.

(B) (Arrow) across the stenosis.
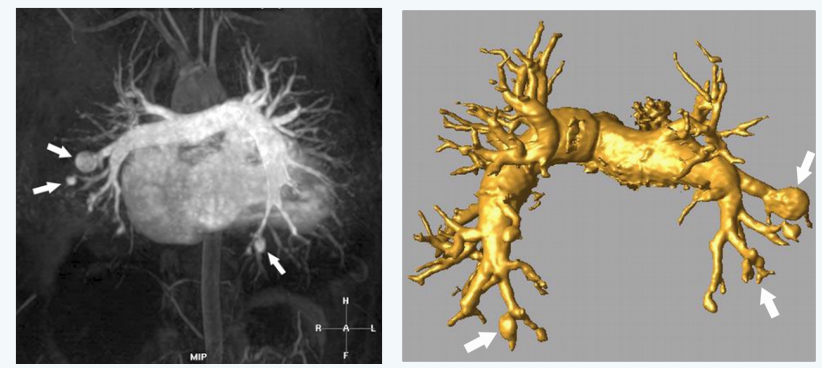

Figure 4: 3D MRA of the pulmonary arteries; Coronal MIP image.

(A) And targeted volume rendering image.

(B) Show mild postenotic dilatation of the proximal portion of pulmonary arteries bilaterally and multiple aneurysms of the segmental branches in both lower lobes and right middle lobe (arrows). Compared to previous CT scans, the diameter of the aneurysm in the middle lobe almost duplicated, the others being unchanged.

The diagnosis of multiple mycotic PAAs due to endocarditis was confirmed. Hemoptysis ceased shortly after hospital admission and therefore, it was attributed to septic pulmonary embolism, as it was presumably the case in his previous admissions. Aggressive surgical or interventional management 
for the PAAs had not been recommended. A new echocardiogram after 30 days administration of the appropriate antibiotic treatment did not show the vegetation of the conduit. The treatment was discontinued and the patient was discharged afebrile. A surgical replacement of the valvular graft was successfully performed one month later. The patient had a straightforward course but he refused a repeat CT scan.

\section{Discussion}

Mycotic PAAs are most commonly seen after right heart endocarditis often associated with congenital heart disease or IV drug use and their detection needs a high index of suspicion in the appropriate clinical setting. Detection of large PAAs is straightforward even with conventional chest CT after i.v contrast administration, [1] but small PAAs need the application of dedicated angiographic techniques using either multi slice CT or MRI. Angiography, however, is felt to be the best diagnostic procedure and can be combined with percutaneous catheter embolization [2] Bronchoscopy and/or inadvertent biopsy of a PAA can lead to massive hemorrhage and death [2]. Owing to their rarity, their natural history is not well documented. It is reported that about one-third of PAAs rupture, causing death but the relationship between size and location of PAAs and their risk of rupture is not known [1]. In a series of cases, more than $50 \%$ of patients have been shown to die as a result of bleeding complications [3] and the need for urgent treatment is established. Treatment for PAAS is directed towards the underlying etiology and can be conservative or invasive. Regression under conservative treatment has been scarcely reported [1]. Definitive therapy involves surgical or endovascular approaches.

\section{Conclusion}

Although endocarditis is a well-known complication of surgical correction of tetralogy of Fallot/pulmonary atresia, the present case has several unique characteristics that add to our knowledge on the natural history of this rare complication. In our patient, small $(<1 \mathrm{~cm}$ in diameter) PAAs developed multi focally in a longtime period during which multiple episodes of septic pulmonary embolism occurred. Endocarditis, although suspected, was never diagnosed and under treatment resulted in chronicity of the infection leading to kachexia with reactive lymphadenopathy, splenomegaly and finally to deterioration of cardiac function. The PAAs remained unchanged for at least two years, but one of them enlarged by $75 \%$, indicating a different behavior. Kocuria varians is an extremely unusual cause of endocarditis [4] with only a couple of cases reported in literature. In the present case the demonstration of the vegetation inside the conduit was confirmed by means of the TTE from the suprasternal projection. This projection is improperly not always performed in daily practice and may be the apparent explanation for the delayed diagnosis of the endocarditis, especially in right sided infected valves or extra cardiac conduits.

\section{References}

1. Wilson TN, Tew K, Taranath A (2008) Multiple mycotic aneurysms of the pulmonary arteries resolving with conservative management: Multi slice CT examination findings J Thoracic Imaging 23(3): 197-201.

2. McLean L, Sharma S, Maycher B (1998) Mycotic pulmonary arterial aneurysms in an intravenous drug user: Can Respir J 5(4): 307-311.

3. Benveniste O, Bruneel F, Bedos JP, Wolff M, Leseche G, et al. (1998) Ruptured mycotic pulmonary artery aneurysm: an unusual complication of right-sided endocarditis. Scand J Infect Dis 30(6): 626629.

4. S Shivaprakasha, K Radhakrishnan, P Kamath, C Jayaprakash, T Shailaja, et al. (2008) Prosthetic valve endocarditis due to Kocuria varians. The Internet Journal of Microbiology 6(1): 1-4.

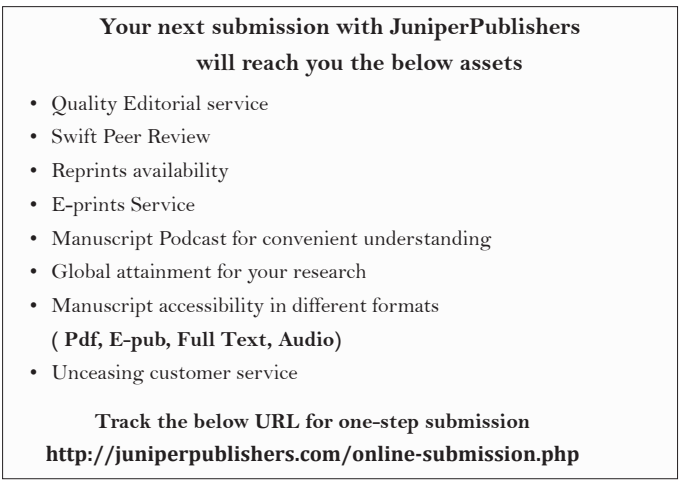

